\title{
Quantitative Electron Probe Microanalysis of Thin Films*
}

\author{
By Yuzo Oda** and Koichi Nakajima**
}

\begin{abstract}
A new method for analysis of thin films by electron microprobe technique is proposed. This method is characterized by the determination of the thickness of thin films together with its chemical compositions.

Results obtained to data through the use of this method have been fairly good; being the accuracy of the order of $10 \%$ in determination of film thickness and of the order of $5 \%$ in quantitative analysis of constituent elements.
\end{abstract}

(Received February 10, 1975)

\section{Introduction}

Surface films thicker than a micron are usually analysed by conventional microprobe technique: the principle of quantitative analysis with the electron probe microanalyser is based on the assumption that the constituent element in a given sample is distributed randomly making solid solution. However, to make a quantitative analysis or estimate of the thickness of a film thinner than the electron penetration depth it is necessary to take account of the behavior of incident electrons and emitted $\mathrm{X}$-rays in the specimen. Some researchers have tried to make use of calibration curves for thickness determination based on the electron excitation curves, and Colby proposed a method of quantitative analysis of thin film with known thickness. Reuter has calculated the ionization function with metals covering a wide range of primary energies, and determined their film thickness.

The purpose of the present study is to discuss the factors involved in quantitative analysis of thin film composed of multicomponents and to establish a method of obtaining its thickness together with the chemical composition.

* This paper was originally published in Japanese in J. Japan Inst. Metals, 37 (1973), 673.

** Toyota Central Research \& Development Laboratories Inc., Tempaku-ku 468, Nagoya.

Trans. JIM

\section{Determination of the Film Thickness and Its Chemical Composition}

The concentration of an element $A$ in a given sample, $C_{A}$, will be deduced by the following relation:

$$
k_{A}=g \cdot C_{A}
$$

where $k_{A}=I_{A}^{S} / I_{A}^{A}$ : the ratio of the intensity, $I_{A}^{S}$, of the characteristic radiation emitted from element $A$ in the sample to the intensity, $I_{A}^{A}$, of the same characteristic radiation emitted from pure element $A$ under the same condition, and $g$ is a correction factor depending on the kind of element, the relative intensity of the characteristic radiation and the geometrical condition of measurement. In practice, it is convenient to use the following relation instead of eq. $(1)^{\prime}$ :

$$
C_{A}=G_{A}^{S}\left(k_{A}\right) \cdot k_{A} .
$$

Correction factor $G_{A}^{S}\left(k_{A}\right)$ is considered to be composed of the main three terms, namely, absorption, atomic number and fluorescence corrections and is given as follows:

$$
G_{A}^{S}\left(k_{A}\right)=G_{A}^{a}\left(k_{A}\right) \cdot G_{A}^{n}\left(k_{A}\right) \cdot G_{A}^{f}\left(k_{A}\right),
$$

where $G_{A}^{a}, G_{A}^{n}$, and $G_{A}^{f}$, are the correction terms for absorption, atomic number and fluorescence, respectively.

The intensity of characteristic X-ray radiation emitted from a constituent element $A$ declines due to the absorption by the sample

1975 Vol. 16 
itself. The correction term for the absorption was given by Castaing $^{(3)}$ as:

$$
\begin{aligned}
& G_{A}^{a}\left(k_{A}\right) \\
& \int_{0}^{\infty} \phi\left(\rho_{\mathrm{A}} x\right) \cdot \exp \left(-\mu_{\mathrm{A}}^{A} \cdot \rho_{\mathrm{A}} \cdot x \cdot \operatorname{cosec} \theta\right) d\left(\rho_{\mathrm{A}} x\right)
\end{aligned}
$$

where $\rho_{A}:$ specific density of pure element $A$ $x$ : depth from the surface

$\theta: \mathrm{X}$-ray take-off angle

$\mu_{A}^{j}$ : mass absorption coefficient of $j$ for the characteristic radiation from element $A$

$\bar{\mu}_{A}: \sum_{j=A}^{N} C_{j} \cdot \mu_{A}^{j}$.

Castaing obtained experimentally the electron excitation function $\phi(\rho x)$ for various element at an operating voltage of $29 \mathrm{kV}$. According to his results, $\phi(\rho x)$ is a function of $\rho x$ but varies only slightly with the atomic number ranging from $Z=13$ to 79 . In general, the average specific density of a given sample may apploximately be given as $\bar{\rho}=\sum_{j=A}^{N} C_{j} \rho_{j}$, provided the sample is in the state of solid solution.

The correction term for the atomic number may be expressed as ${ }^{(4)(5)}$

$$
G_{A}^{n}\left(k_{A}\right)=\frac{\bar{R}}{R_{A}} \cdot \frac{S_{A}}{\bar{S}},
$$

where $R_{A}, \bar{R}$ : back scattering coefficient of $A$ and sample, respectively

$S_{A}, \bar{S}$ : stopping power of element $A$ and specimen.

The fluorescence arises from both the continuous spectrum and the hard component of the characteristic radiation, the contribution of the former being generally smaller than that of the latter ${ }^{(5)}$. The correction for the fluorescence was given by ${ }^{(6)}$

$$
G_{A}^{f}\left(k_{A}\right)=\frac{1}{1+I_{f} / I_{A}^{S}}
$$

\section{Characteristic X-ray Radiation Emitted from the Surface Film on the Substrate}

Characteristic X-ray radiation emitted from the surface film may be divided into the following three groups: (1) those that are excited directly by the incident beam, (2) those that are excited by electrons back-scattered from the substrate, and (3) those that are excited by $\mathrm{X}$-rays from the coexisting heavier elements in the sample (fluorescence).

$\mathrm{X}$-ray intensity emitted from element $A$ in the surface film relating to the above items (1) and (2) is written from eqs. (3) and (4) as:

$$
\begin{aligned}
I_{1}(A)= & C_{A} \cdot \frac{\bar{R}_{F}}{\bar{S}_{F}} \cdot \int_{0}^{\bar{\rho}_{F} t} \phi\left(\bar{\rho}_{F} x\right) \\
& \times \exp \left(-\bar{\mu}_{A}^{F} \cdot \bar{\rho}_{F} \cdot x \cdot \operatorname{cosec} \theta\right) d\left(\bar{\rho}_{F} x\right),
\end{aligned}
$$

where $\bar{\rho}_{F}$ : average specific density of the surface film. $t$ : thickness of film. $\bar{R}_{F}$ and $\bar{S}_{F}$, back scattering coefficient and stopping power of the film, respectively, are obtained by Duncamb and Reed $^{(4)}$ and Springer ${ }^{(5)}$ as functions of atomic number and over voltage ratio.

In general, the constituent elements of the substrate are different from those of the surface film. Therefore, it is necessary to take account of the effect of the electrons back-scattered from the substrate on the surface film. The correction for this is then given by integrating over the range from $\bar{\rho}_{F} t$ to $2 \bar{\rho}_{F} t$ as:

$$
\begin{aligned}
I_{2}(A)= & C_{A} \frac{\bar{R}_{F}}{\bar{S}_{F}}\left(\bar{\eta}_{s}-\bar{\eta}_{F}\right) \cdot \int_{\rho_{F} t}^{2 \bar{\rho}_{F} t} \phi\left(\bar{\rho}_{F} x\right) \\
& \times \exp \left\{-\bar{\mu}_{A}^{F} \cdot \bar{\rho}_{F} \cdot(2 t-x) \cdot \operatorname{cosec} \theta\right\} d\left(\bar{\rho}_{F} x\right)
\end{aligned}
$$

where $\bar{\eta}_{F}=\sum_{j=A}^{N} C_{j} \eta_{j}$ and $\bar{\eta}=\sum_{i=a}^{n} C_{i} \eta_{i}$ shows fraction of electron ${ }^{(7)}$ backscattered from film and substrate, respectively, and $A, B, \ldots, N$ and $a, b, \ldots, n$ denote constituent elements of film and substrate, respectively. X-ray radiation emitted from a given sample relating to the first two items is thus expressed as the sum of eqs. (5) and (6).

The excitation of element $A$ by characteristic radiation from constituent element $j$ which is related to item (3) may be given by multiplying the excitation efficiency, $Q_{A}^{x}$. by the intensity of $\mathrm{X}$-rays as:

$$
I_{3}(A)=C_{A} \cdot \sum_{j=A}^{N} \gamma_{A}^{j} \cdot Q_{A}^{j} \cdot I(j)+C_{A} \sum_{i=a}^{n} \gamma_{A}^{i} \cdot Q_{A}^{i} \cdot I(i)
$$

$$
I(x)=I_{1}(x)+I_{2}(x)
$$

where $\gamma_{A}^{x}=0.5^{(6)}$ when the energy of character- 
istic X-rays in element $A$ is lower than that of the constituent element $j$, and $\gamma_{A}^{x}=0$ when the energy of characteristic X-rays in element $A$ is higher than that of $j \cdot I_{1}(x)$ and $I_{2}(x)$ is given by eqs. (5) and (6), respectively.

In practice, the thickness of the surface film is usually very thin so that the $\mathrm{X}$-ray excitation function relating to item (3) may apploximately be taken as a function of the absorption, because the energy of characteristic X-ray radiation is constant depending on the wave-length. Then, the contribution to the production of $\mathrm{X}$-rays from the thin layer is written by

$$
\begin{array}{r}
\int_{0}^{\bar{\rho}_{F} t} \exp \left(-\bar{\mu}_{A}^{F} \cdot \bar{\rho}_{F} \cdot x \cdot \operatorname{cosec} \theta\right) d\left(\bar{\rho}_{F} x\right) \\
\int_{0}^{\infty} \exp \left(-\mu_{A}^{A} \cdot \rho_{A} \cdot x \cdot \operatorname{cosec} \theta\right) d\left(\rho_{A} x\right) \\
=\left\{1-\exp \left(-\bar{\mu}_{A}^{F} \cdot \bar{\rho}_{F} \cdot t \cdot \operatorname{cosec} \theta\right)\right\} .
\end{array}
$$

By considering eqs. (7) and (8), the fluorescence $\mathrm{X}$-rays from element $A$ in the surface film is expressed as

$$
\begin{aligned}
I_{4}(A)= & C_{A}\left\{\sum_{j=A}^{N} \gamma_{A}^{j} \cdot Q_{A}^{j} \cdot I(j)+\sum_{i=a}^{n} \gamma_{A}^{i} \cdot Q_{A}^{i} \cdot I(i)\right\} \\
& \times\left\{1-\exp \left(-\bar{\mu}_{A}^{F} \cdot t \cdot \operatorname{cosec} \theta\right)\right\} .
\end{aligned}
$$

Then, the correction for the fluorescence is given by

$$
I_{f}^{F}=\frac{I_{4}(A)}{C_{A} \cdot I(A)} .
$$

The fluorescence effect is only an important factor when the coexisting elements are close to element $A$ but larger in atomic number. However, even in such a case its intensity is only about $5 \%$ compared with that excited directly by the incident electrons. Therefore, the fluorescence effect would generally be neglected.

$\mathrm{X}$-ray intensity to be measured practically is finally given as the following equation in comparison with the intensity obtained from pure element:

$$
k_{A}=C_{A} \cdot \frac{1}{G_{A}^{F}\left(k_{A}\right)}
$$

where

$$
k_{A}=\frac{C_{A}}{G_{A}^{F}\left(k_{A}\right)}=\frac{S_{A}}{R_{A}} \cdot \frac{\left\{I_{1}(A)+I_{2}(A)\right\} \times\left(1+I_{f}^{F}\right)}{\int_{0}^{\infty} \phi\left(\rho_{A} x\right) \cdot \exp \left(-\mu_{A}^{A} \cdot \rho_{A} \cdot x \cdot \operatorname{cosec} \theta\right) d\left(\rho_{A} x\right)} .
$$

\section{X-ray Radiation Emitted from the Constituent Element of the Substrate}

X-ray radiation emitted from an element $a$ in the substrate is considered to be composed of the following groups: (1) X-rays excited directly by incident electrons, (2) X-rays excited by electrons scattered by the constituent ele- ments of surface film and (3) X-rays excited by the fluorescence X-rays.

Characteristic $\mathrm{X}$-ray radiation emitted from element $a$ of the substrate is diminished by the surface film as the radiation travels towards the surface by $\exp \left(-\bar{\mu}_{a}^{F} \cdot \bar{\rho}_{F} \cdot t \cdot \operatorname{cosec} \theta\right)$. By assuming that the substrate is of a homogeneous structure the $\mathrm{X}$-rays emitted from element $a$ may be written by

$$
I_{1}(a)=C_{a} \frac{\bar{R}_{s}}{\bar{S}_{s}} \cdot \exp \left(-\bar{\mu}_{a}^{F} \cdot \bar{\rho}_{F} \cdot t \cdot \operatorname{cosec} \theta\right) \cdot \int_{\bar{\rho}_{F t}}^{\infty} \phi\left(\bar{\rho}_{s} x\right) \cdot \exp \left(-\bar{\mu}_{a}^{s} \cdot \bar{\rho}_{s} \cdot \operatorname{cosec} \theta\right) \cdot d\left(\bar{\rho}_{s} x\right)
$$

where the constituent element $a$ exists below the depth, $t$, from the surface.

The constituent elements of the surface film are usually different from those of the substrate.
Therefore, it is necessary to take account of the behavior of the incident electrons at their interface; the correction for this then given by

$$
I_{2}(a)=C_{a} \frac{\bar{R}_{s}}{\bar{S}_{s}} \cdot\left(\bar{\eta}_{s}-\bar{\eta}_{F}\right) \cdot \exp \left(-\bar{\mu}_{a}^{F} \cdot \bar{\rho}_{F} \cdot t \cdot \operatorname{cosec} \theta\right) \cdot \int_{\bar{\rho}_{F t}}^{\infty} \phi\left(\bar{\rho}_{s} x\right) \cdot \exp \left\{-\bar{\mu}_{a}^{s} \cdot \bar{\rho}_{s} \cdot x \cdot \operatorname{cosec} \theta\right\} d\left(\bar{\rho}_{s} x\right)
$$


The fluorescence effect of the element in the given by substrate is given in the same way as eq. (9):

$$
\begin{aligned}
I_{4}(a)= & C_{a} \cdot \exp \left(-\bar{\mu}_{a}^{F} \cdot \bar{\rho}_{F} \cdot t \cdot \operatorname{cosec} \theta\right) \\
& \times\left\{\sum_{j=A}^{N} \gamma_{a}^{j} \cdot Q_{a}^{j} \cdot I(j)+\sum_{i=a}^{n} \gamma_{a}^{i} \cdot Q_{a}^{i} \cdot I(i)\right\} .
\end{aligned}
$$

Finally, the total intensity emitted from element $a$ in the substrate is obtained as the comparison with that of pure element $a$ :

Then, the correction for the fluorescence is

$$
k_{a}=\frac{C_{a}}{G_{a}^{F}\left(k_{a}\right)}=\frac{S_{a}}{R_{a}} \cdot \frac{\left\{I_{1}(a)+I_{2}(a)\left\{\times\left(1+I_{f}^{s}\right)\right.\right.}{\int_{0}^{\infty} \phi\left(\rho_{a} x\right) \cdot \exp \left(-\mu_{a}^{a} \cdot \rho_{a} \cdot x \cdot \operatorname{cosec} \theta\right) d\left(\rho_{a} x\right)}
$$

where

$$
\sum_{j=A}^{N} C_{j}=1, \quad \sum_{i=a}^{n} C_{i}=1 .
$$

Equations (11) and (16) correspond to the number of constituent element of the sample. Using these equations one can calculate the thickness of the surface film together with the concentration of each constituent element. These equations were evaluated for different thickness and chemical compositions in some alloy films, as shown later.

\section{Comparison with Experimental Results}

\section{Estimation of thickness of Fe-Ni alloy films}

The present method of estimating film thickness and its chemical composition was applied to $\mathrm{Fe}-\mathrm{Ni}$ alloy vacuum-deposited on a silica plate.

The specimens were prepared by vacuum deposition of $\mathrm{Fe}$ and $\mathrm{Ni}$ on a silica plate preheated at $350^{\circ} \mathrm{C}$, then annealed at $800^{\circ} \mathrm{C}$ for $30 \mathrm{~min}$. Chemical analysis showed that the composition was $\mathrm{Fe}-6.1 \% \mathrm{Ni}$. The thickness of the film was previously determined by using a multiple beam interferometer, and the result obtained was used as a standard of comparison. The electron probe X-ray microanalyser was operated at $30 \mathrm{kV}$ with $52.5^{\circ}$ in X-ray take-off angle. The effective area irradiated was taken to be about 30 diameter.

Table 1 shows the result of the analysis together with the result obtained by using the multiple beam interferometer, in which the chemical compositions denoted by "measured" are values calculated directly from the raw data, and the values denoted by "corrected" are corrected values by applying the proposed method. It is seen from this table that the value of the film thickness estimated from the proposed method fits neatly to the result obtained from the interferometer.

2. Case of $\mathrm{Ag}-\mathrm{Cu}$ alloy films produced by sputtering on plate of $\mathrm{Fe}-\mathrm{Ni}$ alloy

A plate of $\mathrm{Fe}-\mathrm{Ni}$ alloy polished mechanically was used as the substrate. The concentration of alloying element was determined by chemical analyses and turned out to be $\mathrm{Fe}-25 \% \mathrm{Ni}$, $\mathrm{Ag}-91.5 \% \mathrm{Cu}$ and $\mathrm{Ag}-49.5 \% \mathrm{Cu}$, respectively. The homogeneity of the alloy was confirmed by

\begin{tabular}{|c|c|c|c|c|c|c|}
\hline \multirow{3}{*}{ Specimen } & \multicolumn{4}{|c|}{ Chemical composition } & \multirow{2}{*}{$\begin{array}{c}\text { Thickness } \\
\text { EPMA }\end{array}$} & \multirow{2}{*}{$\begin{array}{c}\text { Thickness } \\
\text { MBI }\end{array}$} \\
\hline & \multicolumn{2}{|c|}{ Measured } & \multicolumn{2}{|c|}{ Corrected } & & \\
\hline & $\mathrm{Fe}$ & $\mathrm{Ni}$ & $\mathrm{Fe}$ & $\mathrm{Ni}$ & & \\
\hline $\mathbf{A}$ & $9.9 \%$ & $0.5 \%$ & $93.6 \%$ & $6.4 \%$ & $1200 \AA$ & $1100 \AA$ \\
\hline
\end{tabular}
using the electron probe microanalyser.

Table 2 lists the results of analyses, in which

Table 1 Thickness determination of Fe-Ni alloy film and its chemical composition. 
Table 2 Thickness determination of $\mathrm{Cu}-\mathrm{Ag}$ alloy films on $\mathrm{Fe}-\mathrm{Ni}$ alloy and their chemical compositions.

\begin{tabular}{|c|c|c|c|c|c|c|}
\hline \multirow{2}{*}{ Secimen } & & \multicolumn{4}{|c|}{ Chemical composition } & \multirow{2}{*}{$\begin{array}{l}\text { Thickness } \\
\text { MBI }\end{array}$} \\
\hline & & $\mathrm{Ag}$ & $\mathrm{Cu}$ & $\mathrm{Fe}$ & $\mathrm{Ni}$ & \\
\hline \multirow{3}{*}{ A } & Measured & $56.2 \%$ & $6.1 \%$ & $26.1 \%$ & $8.6 \%$ & $4800 \AA$ \\
\hline & Corrected I & $62.9 \%$ & $5.8 \%$ & $26.2 \%$ & $8.3 \%$ & EPMA \\
\hline & Corrected II & $90.8 \%$ & $9.2 \%$ & $75.3 \%$ & $24.7 \%$ & $4700 \AA$ \\
\hline \multirow{3}{*}{ B } & Measured & $2.3 \%$ & $1.8 \%$ & $76.8 \%$ & $24.4 \%$ & MBI \\
\hline & Corrected I & $2.9 \%$ & $2.3 \%$ & $72.9 \%$ & $29.3 \%$ & $400 \AA$ \\
\hline & Corrected II & $51.0 \%$ & $49.0 \%$ & $74.3 \%$ & $25.7 \%$ & $\begin{array}{l}\text { EPMA } \\
420 \AA\end{array}$ \\
\hline
\end{tabular}

"corrected I" is the result corrected by using conventional microprobe technique and "corrected II" the result after the correction by using the proposed method. It is seen from this table that the results obtained agree well with those obtained by the interferometer, within the range of about $10 \%$.

\section{Thin layer of $\mathrm{Sn}$ in $\mathrm{Al}-\mathrm{Sn}$ bearing metal}

The proposed method may be applied to films as thin as several ten $\AA$, depending on the kind of constituent elements. The present authors $^{(8)}$ have investigated the surface film of Al-6wt $\% \mathrm{Sn}$ alloy formed during friction. Solubility limit of $\mathrm{Sn}$ in $\mathrm{Al}$ is known to be $0.04 \%$ (weight $^{(9)}$. However, electron microprobe analysis of the surface after friction showed to turn out to be $0.18 \%$, showing a homogeneous distribution. This result suggests a formation of thin film of Sn on the surface during the friction. The film thickness was determined by applying this method, and the result showed an existence of a very thin layer of $\mathrm{Sn}$, the thickness being of the order of several ten $\AA$.

\section{Summary}

A new method for the determination of film thickness together with its chemical composition has been developed.

This method was applied to analyse various thin metal and alloy films. It was shown that films thinner than the penetration depth of incident electrons could be analysed with sufficient accuracy: the relative error is written about $10 \%$ for their thickness determination and about $5 \%$ for the determination of their chemical composition.

\section{REFERENCES}

(1) J. W. Colby: Advance in X-ray analysis, Plenum Press, New York., 11 (1967), 287.

(2) W. Reuter: X-ray Optics and Microanalysis, University of Tokyo Press, (1972), p. 121.

(3) R. Castaing: Advance in electronics and electron physics, Academic Press, (1980), p. 317.

(4) P. Duncamb and S. J. B. Reed: Quantitative electron probe microanalysis, National Bureau of Standard Special Publication, (1966), p. 133.

(5) G. Springer: X-ray Optics and Microanalysis, Boulerard Saint-German Paris, (1965), p. 296.

(6) S. B. J. Reed: British J. Appl. Phys., 16 (1965), 913.

(7) H. E. Bishop: Optique des Rayons-Xet Microanalysis, Herman, Paris, (1967).

(8) Y. Oda, Y. Mizutani and K. Nakajima: J. Japan Inst. Metals, 36 (1972), 859.

(9) R. P. Elliot: Constitution of Binary Alloys, First Supplement, McGraw-Hill Book Co., New York, (1965), p. 56. 